\title{
$\Omega$
}

Pierantonio Laveneziana ${ }^{1,2}$, Marie-Cécile Niérat ${ }^{1}$,

[1]

'Sorbonne Université, INSERM, UMRS1158 Neurophysiologie respiratoire expérimentale et clinique, Paris, France. ${ }^{2} \mathrm{AP}-\mathrm{HP}$, Groupe Hospitalier Pitié-Salpêtrière Charles Foix, Service des Explorations Fonctionnelles de la Respiration, de l'Exercice et de la Dyspnée du Département R3S, Paris, France. ${ }^{3}$ Dipartimento di Elettronica, Informazione e Bioingegneria, Politecnico di Milano, Milan, Italy.

\section{A case of unexplained dyspnoea: when lung function testing matters!}

\section{Lung fuction corner}

"Lung function corner" articles in Breathe present the results of a lung function test and the authors then debate the interpretation, including potential controversies and background from the literature. As section editors of this newly created section of Breathe, we felt it was important to write the first article, which highlights the usefulness of lung function testing in guiding clinical diagnosis especially in difficult cases such the one we discuss here.

\section{Case history}

A 36-year-old physically active man is referred for dyspnoea on exertion such as climbing stairs (three floors), playing soccer, during diving and when speaking for a long time.

He revealed that his symptoms appeared a few days after a cervical manipulation by a physiotherapist due to cervical pain on the left side of his neck. He is a current smoker (5 pack-years). He suffered from Parsonage-Turner syndrome (a brachial plexus neuropathy of uncertain cause characterised by rapid onset of severe pain in the shoulder and arm, that usually resolves in most of the affected individuals) accompanied by right arm deficit in 1996 with no long-term consequences or sequelae. His current body mass index is $26 \mathrm{~kg} \cdot \mathrm{m}^{-2}$. His Medical Research Council score for dyspnoea is 1-2. He admitted breathlessness while lying in the supine position (orthopnoea) and bending down or over to pick something up, e.g. to tie shoelaces or to garden (antepnoea) but denied breathlessness while lying on the left or right side (lateropnoea). On a recent resting echocardiography, left ventricular ejection fraction was normal. Physical examination during spontaneous resting breathing was strictly normal: chest movement was equally bilateral, there was no abdominal paradox, or inspiratory neck muscle anomalies, or alterations on pulmonary and cardiac auscultation.

\section{Questions}

What is the primary cause of his unexplained dyspnoea? Would pulmonary function testing (PFT) including respiratory muscle evaluation be an appropriate means to answer these questions?
Cite as: Laveneziana $P$, Niérat M-C, LoMauro A, et al. A case of unexplained dyspnoea: when lung function testing matters! Breathe 2018; 14: 325-332.

@ERSpublications

Diverse methods are available for assessment of the respiratory muscles; the technique used should be tailored to the question posed. http://ow.ly/ChbX30m91bt 


\section{Answers}

A PFT including respiratory muscle evaluation at this stage would be an appropriate means to identify the cause of dyspnoea.

Table 1 present the results from spirometry and lung volumes in an upright sitting and supine position.

PFT in an upright sitting position clearly showed a restrictive ventilatory defect as characterised by a reduction in TLC below the fifth percentile of the predicted value (LLN), and a normal FEV1/VC [1] To elucidate the origin of this restrictive ventilatory defect (chest wall or neuromuscular disorders), diffusing capacity of the lung for carbon monoxide ( $D \mathrm{LCO})$, arterial blood gas analysis and a respiratory muscle evaluation were performed. Arterial blood gas analysis was normal. D LCO was slightly reduced in absolute value but the transfer coefficient of the lung for carbon monoxide (KCO) was slightly increased, suggesting respiratory muscle or chest wall disease, rather than microvascular or lung parenchymal involvement, therefore making the functional orientation switch more towards neuromuscular disorders. Respiratory muscle evaluation revealed a weakness of the inspiratory muscles (table 2), with a MIP, MEP and SNIP all reduced to $55 \%, 71 \%$ and $50 \%$ predicted, respectively [2, 3].

In the presence of potential respiratory muscle weakness, a simple functional test available in
PFT laboratories to orient towards diaphragmatic dysfunction is the evaluation of VC in the supine position (table 1). A difference of $35 \%$ was found between the VC measured in an upright sitting versus supine position. This finding made us suspect a diaphragmatic dysfunction due to neuralgic amyotrophy (given the history of acute shoulder or neck pain followed by dyspnoea), which was confirmed by the invasive standard procedure involving electrical and magnetic phrenic nerve stimulation with transdiaphragmatic pressure. Of note, on a chest radiograph requested after the lung function testing, he was found to have an elevated right hemidiaphragm. The computed tomography (CT) scan showed no lesions of the right phrenic nerve. The patient was transferred to the pulmonology department for a complete workup. Follow-up muscle evaluations between 1 and 3 years after initial referral showed complete recovery of diaphragmatic function in our patient. Diaphragmatic strength returned very slowly.

\section{State of the art and controversial issues}

Patients with unilateral diaphragmatic paralysis are usually asymptomatic but may present with exertional dyspnoea and, occasionally, with orthopnoea. Patients with bilateral diaphragmatic

Table 1 Resting PFT in an upright sitting and supine position

\begin{tabular}{|c|c|c|c|c|c|c|c|}
\hline & \multicolumn{5}{|c|}{ Upright sitting position } & \multicolumn{2}{|c|}{ Supine position } \\
\hline & Measured & LLN & ULN & $\begin{array}{l}\text { Reference } \\
\text { (mean value) }\end{array}$ & $\begin{array}{l}\% \text { of } \\
\text { reference }\end{array}$ & Measured & $\begin{array}{l}\% \text { of } \\
\text { reference }\end{array}$ \\
\hline VC L & 4.13 & 4.33 & 6.17 & 5.25 & $79 \%$ & 2.70 & $51 \%$ \\
\hline TLC L & 5.79 & 6.07 & 8.37 & 7.22 & $80 \%$ & 4.17 & $58 \%$ \\
\hline FRC L & 2.39 & 2.43 & 4.41 & 3.42 & $70 \%$ & 1.65 & $48 \%$ \\
\hline IC L & 3.40 & & & 3.47 & $98 \%$ & 2.52 & $73 \%$ \\
\hline RV L & 1.65 & 1.24 & 2.58 & 1.91 & $87 \%$ & 1.47 & $77 \%$ \\
\hline ERV L & 0.73 & & & 1.73 & $42 \%$ & 0.18 & $10 \%$ \\
\hline RV/TLC \% & 29 & 19 & 37 & 28 & $102 \%$ & 35 & $126 \%$ \\
\hline FEV 1 L & 3.07 & 3.32 & 5.00 & 4.16 & $74 \%$ & & \\
\hline FVC L & 4.04 & 4.03 & 6.03 & 5.03 & $80 \%$ & & \\
\hline FEV1/VC \%\# & 74 & 69 & 92 & 81 & $94 \%$ & & \\
\hline PEF L·- $\mathbf{s}^{-1}$ & 8.11 & 7.59 & 11.57 & 9.58 & $85 \%$ & & \\
\hline FEF25\% L-s ${ }^{-1}$ & 6.93 & 5.44 & 11.06 & 8.25 & $84 \%$ & & \\
\hline FEF50\% L-s $\mathrm{s}^{-1}$ & 3.52 & 3.14 & 7.48 & 5.31 & $66 \%$ & & \\
\hline FEF75\% L's $\mathbf{s}^{-1}$ & 0.91 & 1.11 & 3.67 & 2.39 & $38 \%$ & & \\
\hline
\end{tabular}

LLN: lower limit of normality; ULN: upper limit of normality; VC: vital capacity; TLC: total lung capacity; FRC: functional residual capacity; IC: inspiratory capacity; RV: residual volume; ERV: expiratory reserve volume; FEV1: forced expiratory volume in $1 \mathrm{~s}$; FVC: forced vital capacity; PEF: peak expiratory flow; FEFx\%: forced expiratory flow measured after $\mathrm{x} \%$ of the FVC has been exhaled. \#: spirometric evidence of an obstructive ventilatory defect as defined by a reduced FEV1/VC ratio less than the fifth percentile of the predicted value [1]. 


\begin{tabular}{|c|c|c|c|c|c|}
\hline & Measured & LLN & ULN & $\begin{array}{c}\text { Reference } \\
\text { (mean value) }\end{array}$ & $\%$ of reference \\
\hline MIP sustained for $1 \mathrm{~s}$ (at RV) $\mathrm{cmH}_{2} \mathrm{O}$ & 68 & 80 & 168 & 124 & $55 \%$ \\
\hline MEP sustained for $1 \mathrm{~s}$ (at TLC) $\mathrm{cmH}_{2} \mathrm{O}$ & 166 & 149 & 317 & 233 & $71 \%$ \\
\hline \multicolumn{6}{|l|}{ MEP at peak value (at TLC) $\mathrm{cmH}_{2} \mathrm{O}$} \\
\hline
\end{tabular}

MIP: maximal inspiratory pressure; MEP: maximal expiratory pressure; SNIP: sniff nasal inspiratory pressure. \#: reference values taken from $[2,3]$.

paralysis or severe diaphragmatic weakness are usually symptomatic and may have considerable dyspnoea on exertion, when lying in the supine position, when immersed in water above their waist, or even at rest. Once suspected, diaphragmatic dysfunction can be confirmed by several tests. Decisions about workup are generally made on the basis of the invasiveness and availability of testing. Definitive diagnosis of diaphragm dysfunction can be obtained by phrenic nerve stimulation combined with diaphragmatic electromyography and/or twitch transdiaphragmatic pressure (twitch $P$ di) measurement.

Simple and noninvasive PFTs, especially measurements of upright and supine VC which depend on activation of both inspiratory and expiratory muscles [4], are readily available, and may support or refute the suspicion of respiratory muscle dysfunction, especially of the diaphragm (figures 1 and 2) [4-6]. However, the major limitation of upright VC is that MIP decreases earlier than upright VC, this occurs especially in neuromuscular diseases, thus making the suspicion difficult and postponing the correct diagnosis if relying exclusively on upright VC. Unilateral diaphragm weakness is usually associated with a mild decrease in VC, to $\sim 75 \%$ of the predicted value $[7,8]$, with a further $10-20 \%$ decrease in the supine position $(15 \%$ representing twice the coefficient of variation of the measure that could be considered as the ULN) (figure 2) [8], while FRC and TLC are usually preserved in the seated position $[7,8]$, but are sometimes
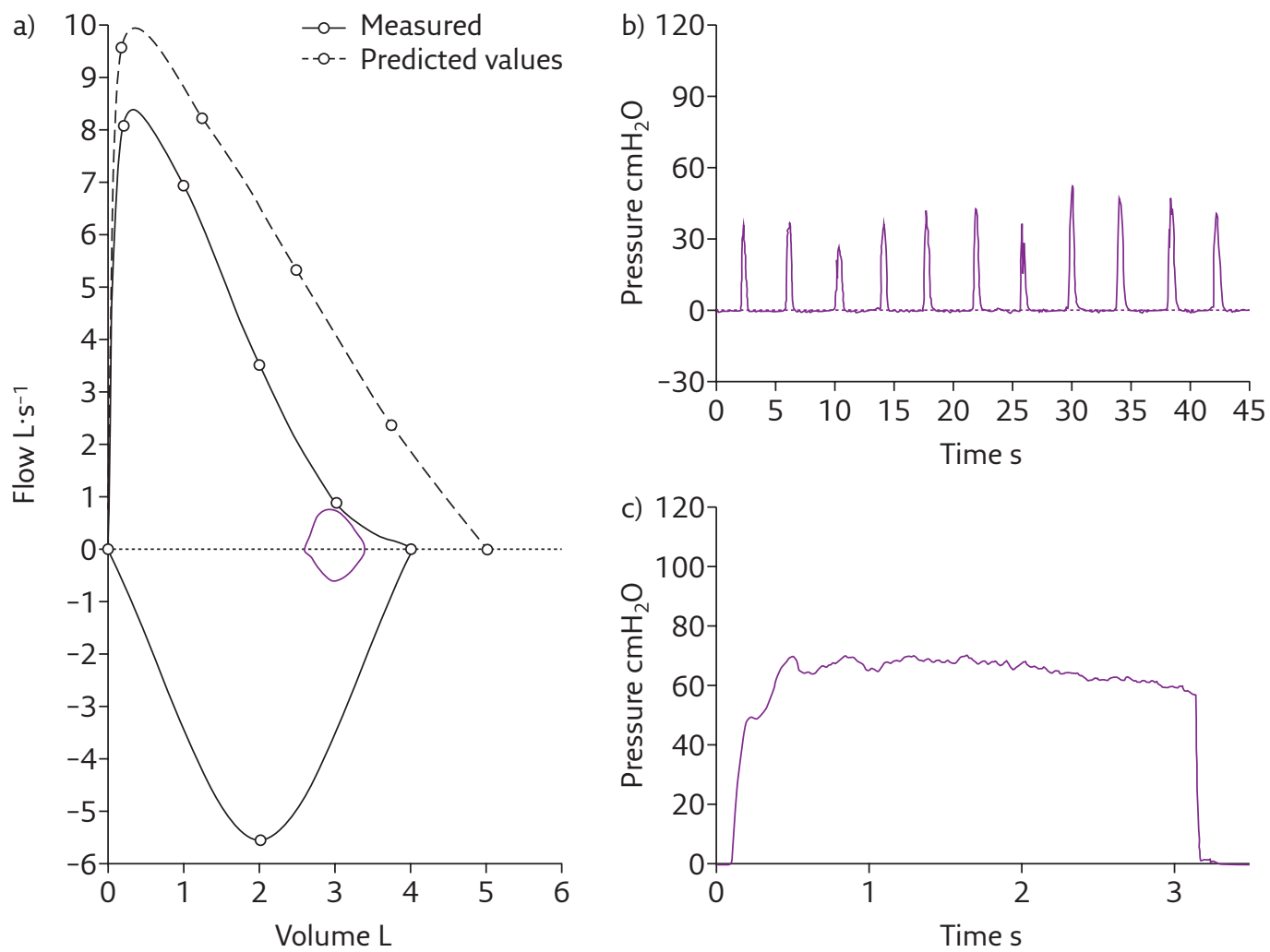

Figure 1 a) Maximal (outer black loop) and tidal (inner purple loop) flow-volume loops at rest in our patient. The predicted values loop is shown as a dashed profile. b) SNIP traces at rest in our patient. c) MIP traces at rest in our patient. Please refer to the text for more details. 


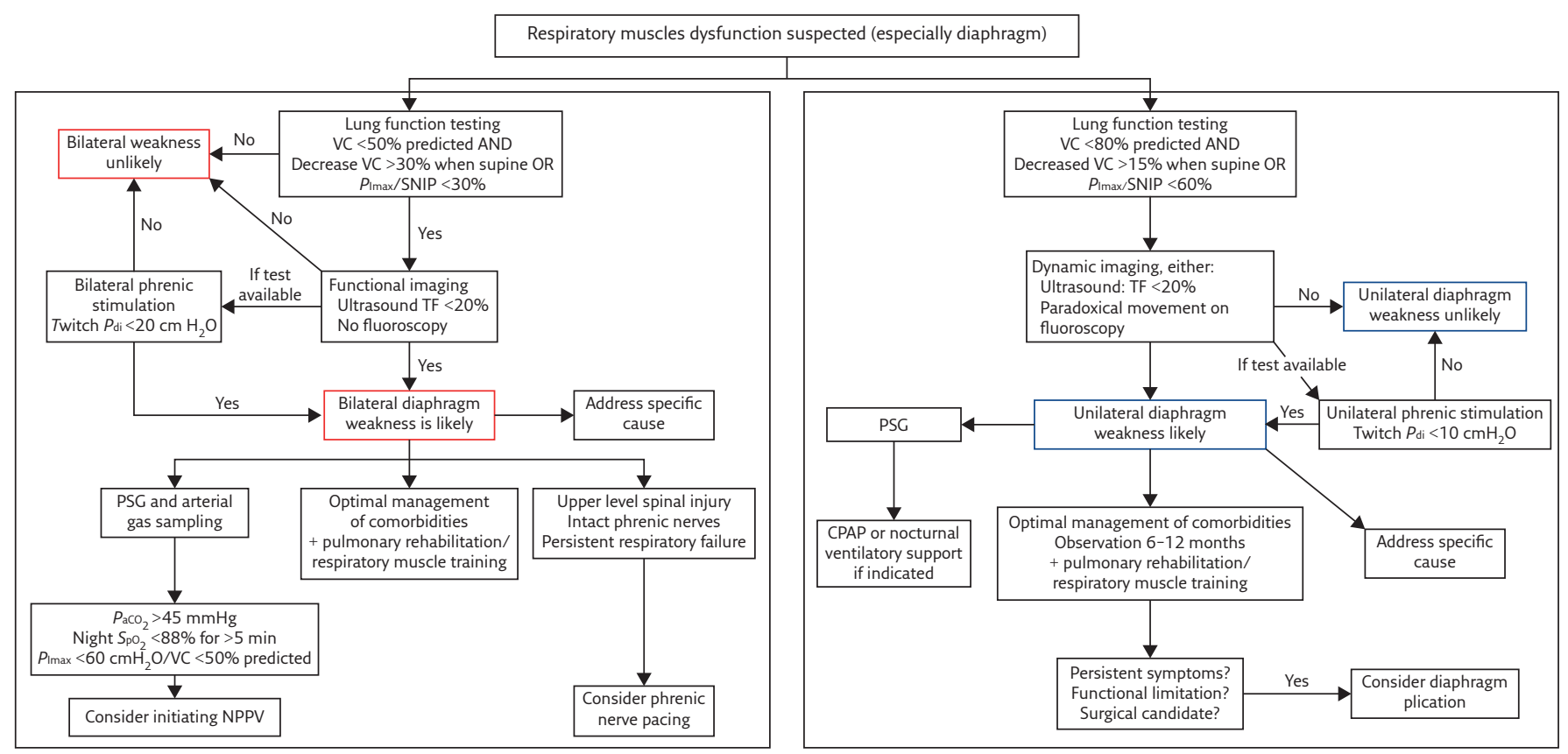

Figure 2 Current practice on the suspicion of respiratory muscle dysfunction (especially of the diaphragm), outside the intensive care setting. The figure describes how a clinician or physiologist suspects and treats respiratory muscle dysfunction (especially unilateral and bilateral diaphragm weakness), outside the intensive care setting. PImax: maximal inspiratory pressure; TF: thickening fraction of the diaphragm; PSG: polysomnography; CPAP: continuous positive airway pressure; NPPV: noninvasive positive pressure ventilation; $\mathrm{PaCO}_{2}$ : arterial carbon dioxide tension; $\mathrm{SpO}_{2}$ : arterial oxygen saturation measured by pulse oximetry. Please refer to the text for more details.

reduced in severe cases [9]. In bilateral diaphragm weakness, VC usually reaches mean values of $\sim 50 \%$ predicted and can further decrease by $30-50 \%$ when supine [10]. A normal supine VC makes the presence of clinically significant diaphragmatic weakness unlikely. TLC can also be reduced in the seated position $(70-79 \%$ of the predicted value for mildly restriction and up to $30-50 \%$ of the predicted value in moderate-to-severe restriction) [9], while $\mathrm{RV}$ can be either increased or decreased or normal in the seated position (with a $\mathrm{RV}$ ranging from $<50$ to $>150 \%$ predicted) [11]. Of note, the magnitude of the fall in VC in the supine position has been shown to be correlated to sniff $P$ di in this population [11]. The mechanism related to the reduction in supine VC is the cephalad displacement of abdominal contents in concert with ineffective activity of the accessory inspiratory muscles. A significant reduction in VC at diagnosis as well as its change or rate of decline over time are generally recognised as being among the criteria for initiating noninvasive ventilation [12, 13], and as being predictive of sleep disordered breathing, respiratory failure, prognosis, evolution and response to treatment to a lesser extent in a wide range of neuromuscular disorders [14, 15], especially amyotrophic lateral sclerosis, with good sensitivity (80-95\%) but variable specificity (50-90\%) [16]. Conventionally, inspiratory and expiratory muscle strength has been evaluated by MIP and MEP at the mouth sustained for $1 \mathrm{~s}$ during a maximal static manoeuvre against a closed shutter [17]. However, MIP and MEP are volitional tests and are poorly reproducible with a coefficient of variation of $25 \%$ [17]. A sniff manoeuvre is more natural and easier to perform. Pressure measured during sniff manoeuvres in the nostril (SNIP) are more reproducible and useful measures of global inspiratory muscle strength. Nevertheless, sniff manoeuvres are also effort-dependent tests and are difficult to interpret in ill or dyspnoeic patients performing submaximal efforts.

The gold standard method of evaluating the mechanical function of the major inspiratory muscles, i.e. the diaphragm, is measurement of the pressure generated by diaphragm contraction in response to phrenic nerve stimulation [17], during which this pressure can be assessed using the difference between the oesophageal and the gastric pressures (twitch $P$ di). As mentioned previously, the best way to quantify diaphragm contractility is to monitor this pressure during a sniff manoeuvre (sniff $P$ di) or during maximal inspiratory efforts against a closed airway ( $P$ di,max). On one hand these manoeuvres are largely effort dependent and are therefore variable. On the other hand, stimulation of the phrenic nerves generates a non-volitional contraction of the diaphragm. It is worth noting that while transcutaneous electrical phrenic nerve stimulation can be applied at the level of the neck (uni- or bilaterally), it has the disadvantage of being technically difficult in patients with obesity or anatomical variations of the phrenic nerve trajectories and is sometimes uncomfortable and painful. Magnetic stimulation of the phrenic nerves is less painful, can be applied bilaterally at the level of the cervical spine or uni or bilaterally at the neck, is reproducible in normal subjects [17] and is easy to perform. Overall, while phrenic nerve 
stimulation techniques are relatively easy tests that have been widely adopted for evaluating diaphragm function, they have the disadvantages of being timeconsuming, of requiring considerable expertise and specialised equipment, and, as such, are poorly adapted to routine clinical practice [17]. Other novel means of evaluating diaphragm function will therefore be welcome.

\section{Points to emphasise}

An important point to emphasise is the absence of clearly defined lower limits of normality for measures of global inspiratory and specifically diaphragmatic strength. It has long been accepted that a MIP of $-80 \mathrm{cmH}_{2} \mathrm{O}$ in men and $-70 \mathrm{cmH}_{2} \mathrm{O}$ in women usually excludes clinically important inspiratory muscle weakness and that a normal MEP with a low MIP may suggest isolated diaphragmatic weakness. A more invasive, yet voluntary, measure used to estimate the strength of the diaphragm is the maximal transdiaphragmatic pressure ( $P$ di,max) obtained by inserting oesophageal and gastric balloon-catheters, with normal values widely ranging between 60 and $240 \mathrm{cmH}_{2} \mathrm{O}$. It is generally accepted that absolute values of $P$ di,max $\geq 80 \mathrm{cmH}_{2} \mathrm{O}$ in men and $\geq 70 \mathrm{cmH}_{2} \mathrm{O}$ in women are generally thought to exclude clinically significant diaphragm weakness. The $P$ di measured during a sniff manoeuvre (sniff $P$ di) also reflects diaphragm strength and may therefore represent an appealing alternative because it is much simpler to perform than $P$ di,max. In clinical practice, sniff $P$ di maximal values $>100 \mathrm{cmH}_{2} \mathrm{O}$ in males and $>80 \mathrm{cmH}_{2} \mathrm{O}$ in females are unlikely to be associated with clinically significant diaphragm weakness. Oesophageal pressure ( $P_{\text {oes }}$ ) or pressure measured in one nostril obtained during a sniff manoeuvre (sniff Poes and SNIP, respectively) reflect the integrated pressure of the inspiratory muscles on the lungs, and values numerically greater than $-70 \mathrm{cmH}_{2} \mathrm{O}$ in males or $-60 \mathrm{cmH}_{2} \mathrm{O}$ in females are also unlikely to be associated with significant inspiratory muscle weakness [17]. However, these measures are not specifically of diaphragm action but instead reflect the integrated pressure of all the inspiratory muscles involved in the sniff, therefore making it difficult to detect the presence of weakness of one or more of the inspiratory muscles. Generally, and broadly speaking, unilateral and bilateral diaphragm paralysis can be expected to decrease MIP or SNIP in the ranges of $60 \%$ [8] and $<30 \%$ [10] of the predicted values, respectively (figure 2 and table 3). An important point to bear in mind is that the agreement between SNIP and MIP is variable; therefore, it has been suggested that these variables should be regarded as complementary and not interchangeable in the evaluation of inspiratory muscle weakness. MEP is generally preserved unless the underlying disease involves both the inspiratory and expiratory muscles (e.g. muscular dystrophy). It is worth noting that MEP may be found to be mildly reduced, in the range of $70-80 \%$ of the predicted value, simply due to a restriction-related reduction of TLC which may affect the optimal length-tension relationships of the expiratory muscles at that volume.

However, these values may be greatly impacted by the presence of underlying obstructive or restrictive lung disease [7] or obesity, especially if severe [18], and during acute exacerbations of the underlying disease, as is the case for chronic obstructive pulmonary disease (COPD) [19]. The case of COPD merits some clarification: the occurrence of progressive lung hyperinflation

Table 3 Main differences between unilateral and bilateral diaphragmatic paralysis

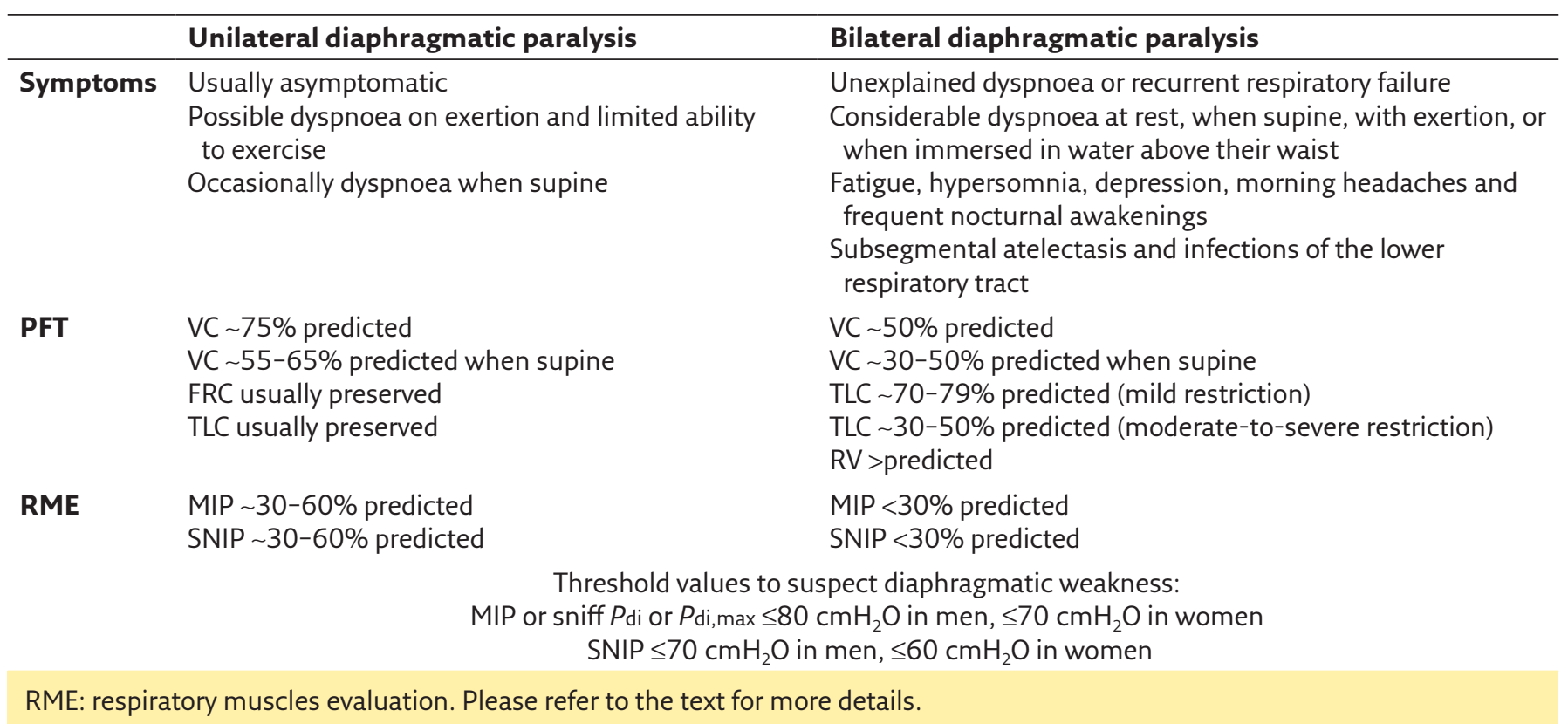




\section{Self-evaluation questions}

1. Concerning the evaluation of respiratory dysfunction of neuromuscular patients, which of the following is/are correct?

a) The residual volume may be either increased or decreased or normal

b) MIP decreases earlier than VC

c) Night-time recording of transcutaneous carbon dioxide is useful for detecting nocturnal hypoventilation

d) The reduction of VC in dorsal decubitus suggests diaphragmatic dysfunction

2. Regarding neuromuscular diseases, which of the following is correct?

a) Dyspnoea is an early sign of hypoventilation

b) Orthopnoea and poor sleep quality only appear in the very advanced stages

c) A MIP of $-80 \mathrm{cmH}_{2} \mathrm{O}$ in men and $-70 \mathrm{cmH}_{2} \mathrm{O}$ in women usually excludes clinically important inspiratory muscle weakness

d) TLC is always reduced

e) None of the above

3. Which one of the following pulmonary function variables best reflects the strength of the diaphragm?
a) MIP
b) MEP
c) FEV1
d) VC
e) Peak expiratory flow

a suggestion that structural adaptations in the inspiratory muscles, particularly in the diaphragm, cause them to become resistant to fatigue $[22,23]$.

Another point to emphasise here is that all the measures of global inspiratory or specifically diaphragm function are also influenced by sex, age, posture, lung volume at which they are realised, and the type of mouthpiece used [17]. It is also worth noting that their limits of normality widely vary between populations of different origin, therefore it is a common recommendation, when assessing a given individual, to use the reference values obtained from the individual's population of origin. A definitive diagnosis, however, is reached only by assessing phrenic nerve stimulation and twitch $P$ di: a twitch $P$ di $>10 \mathrm{cmH}_{2} \mathrm{O}$ with unilateral phrenic nerve stimulation or $>20 \mathrm{cmH}_{2} \mathrm{O}$ with bilateral phrenic nerve stimulation rules out clinically significant diaphragm weakness [17]

\section{Conclusions and future directions}

Diaphragmatic dysfunction is an underdiagnosed cause of unexplained dyspnoea, such as in our patient. It is very difficult to quantify the prevalence of diaphragmatic dysfunction among patients with unexplained breathlessness; the data in the literature are scant and sometimes difficult to interpret. Of course, this would absolutely be an important avenue for future research.

The suspicion of diaphragmatic dysfunction can be supported or refuted by simple lung and respiratory muscle function tests, as was the case in our patient. A suggested diagnostic and therapeutic algorithm for unilateral and bilateral diaphragm weakness is proposed in Figure 2 and explained in greater detail in an ERS statement currently being prepared on this topic. The definitive diagnosis of diaphragm dysfunction requires phrenic nerve stimulation and twitch $P_{\mathrm{di}}$ measurement, which involves highly experienced physiology specialists and may, therefore, not be available at some institutions. This may cause a

\section{Key points}

- Diaphragmatic dysfunction is an underdiagnosed cause of dyspnoea and should always be considered in the differential diagnosis of unexplained dyspnoea.

- Patients with unilateral diaphragmatic paralysis are usually asymptomatic but may have dyspnoea on exertion and limited ability to exercise.

- Once suspected, unilateral or bilateral diaphragmatic weakness can be evaluated using the simple lung and respiratory muscle function tests, readily available in pulmonary function testing laboratories.

- The definitive diagnosis of diaphragm dysfunction can be obtained by phrenic nerve stimulation combined with diaphragmatic electromyography and/or twitch transdiaphragmatic pressure (twitch $P$ di) measurement.

- However, these specific techniques require highly experienced physiology or ultrasonography specialists and may, therefore, not be available in some institutions or intensive care units. This may cause a significant delay in the diagnosis of these conditions.

- New approaches to provide simple, noninvasive, non-contact, early and easy-to-access procedures to diagnose diaphragmatic dysfunction are needed. Optoelectronic plethysmography, magnetic resonance imaging, respiratory muscle mechanomyogram and structured light plethysmography are promising new techniques. 
significant delay in diagnosis of these conditions. As such, new approaches to provide simple, noninvasive and easy-to-access procedures to diagnose diaphragmatic dysfunction are needed. Optoelectronic plethysmography (OEP) is an established technique that allows measurement of the variations in the volume of the chest wall and its compartments during breathing $[24,25]$. OEP has also been adopted to study several neuromuscular disorders [26-28]. Magnetic resonance imaging [29], ultrasonography $[19,30]$ and respiratory muscle mechanomyogram [31] are being increasingly used to evaluate an altered function of the diaphragm.
Another emerging imaging tool is structured light plethysmography (SLP). SLP is a non-contact, noninvasive, easy to use method of assessment of breathing pattern and thoracoabdominal behaviour during spontaneous tidal breathing that allows the measurement of ventilatory activity through the stereoscopic analysis of respiratory-related distortions of a black and white checked pattern projected onto the chest wall and abdomen [32]. SLP has been validated in healthy subjects and in patients [32]. Further research is required to extend these observations, but these preliminary observations are promising.

\section{Author contributions}

All authors contributed to the content, writing and final approval of the manuscript.

\section{Conflict of interest}

P. Laveneziana reports personal fees from Novartis France and from Boehringer France, outside the submitted work. M-C. Niérat has nothing to disclose. A. LoMauro has nothing to disclose. A. Aliverti reports that he has a patent for optoelectronic plethysmography licensed to BTS Bioengineering.

\section{References}

1. Pellegrino R, Viegi G, Brusasco V, et al. Interpretative strategies for lung function tests. Eur RespirJ 2005; 26: 948-968.

2. Black LF, Hyatt RE. Maximal respiratory pressures: Normal values and relationship to age and sex. Am Rev Respir Dis 1969; 99: 696-702.

3. Uldry C, Fitting JW. Maximal values of sniff nasal inspiratory pressure in healthy subjects. Thorax 1995; 50: 371-375.

4. De Troyer A, Borenstein S, Cordier R. Analysis of lung volume restriction in patients with respiratory muscle weakness. Thorax 1980; 35: 603-610

5. Lechtzin N, Wiener CM, Shade DM, et al. Spirometry in the supine position improves the detection of diaphragmatic weakness in patients with amyotrophic lateral sclerosis. Chest 2002; 121: 436-442.

6. Varrato J, Siderowf A, Damiano P, et al. Postural change of forced vital capacity predicts some respiratory symptoms in ALS. Neurology 2001; 57: 357-359.

7. Lisboa C, Pare PD, Pertuze J, et al. Inspiratory muscle function in unilateral diaphragmatic paralysis. Am Rev Respir Dis 1986; 134: 488-492.

8. Laroche CM, Mier AK, Moxham J, et al. Diaphragm strength in patients with recent hemidiaphragm paralysis. Thorax 1988 43: 170-174.

9. Hart N, Nickol AH, Cramer D, et al. Effect of severe isolated unilateral and bilateral diaphragm weakness on exercise performance. Am J Respir Crit Care Med 2002; 165: 1265-1270.

10. Laroche CM, Carroll N, Moxham J, et al. Clinical significance of severe isolated diaphragm weakness. Am Rev Respir Dis 1988; 138: 862-866.

11. Mier-Jedrzejowicz A, Brophy C, Moxham J, et al. Assessment of diaphragm weakness. Am Rev Respir Dis 1988; 137: 877-883

12. Clinical indications for noninvasive positive pressure ventilation in chronic respiratory failure due to restrictive lung disease, COPD, and nocturnal hypoventilation-a consensus conference report. Chest 1999; 116: 521-534.

13. Johnson EM, Roberts M, Mozaffar T, et al. Pulmonary function tests (maximum inspiratory pressure, maximum expiratory pressure, vital capacity, forced vital capacity) predict ventilator use in late-onset Pompe disease. Neuromuscul Disord 2016; 26: 136-145
14. Phillips MF, Quinlivan RC, Edwards RH, et al. Changes in spirometry over time as a prognostic marker in patients with Duchenne muscular dystrophy. Am J Respir Crit Care Med 2001; 164: 2191-2194

15. Van der Beek NA, Hagemans ML, Reuser AJ, et al. Rate of disease progression during long-term follow-up of patients with late-onset Pompe disease. Neuromuscul Disord 2009; 19 : 113-117.

16. Ragette R, Mellies U, Schwake C, et al. Patterns and predictors of sleep disordered breathing in primary myopathies. Thorax 2002; 57: 724-728.

17. American Thoracic Society/European Respiratory Society. ATS/ERS statement on respiratory muscle testing. Am J Respir Crit Care Med 2002; 166: 518-624.

18. Steier J, Lunt A, Hart N, et al. Observational study of the effect of obesity on lung volumes. Thorax 2014; 69: 752-759.

19. Antenora F, Fantini R, lattoni A, et al. Prevalence and outcomes of diaphragmatic dysfunction assessed by ultrasound technology during acute exacerbation of COPD: a pilot study. Respirology 2017; 22: 338-344.

20. Similowski T, Yan S, Gauthier AP, et al. Contractile properties of the human diaphragm during chronic hyperinflation. N Engl J Med 1991; 325: 917-923.

21. Polkey MI, Kyroussis D, Hamnegard $\mathrm{CH}$, et al. Diaphragm strength in chronic obstructive pulmonary disease. Am J Respir Crit Care Med 1996; 154: 1310-1317.

22. Mador MJ, Kufel TJ, Pineda LA, et al. Diaphragmatic fatigue and high-intensity exercise in patients with chronic obstructive pulmonary disease. Am J Respir Crit Care Med 2000; 161: 118-123.

23. Orozco-Levi M, Gea J, Lloreta JL, et al. Subcellular adaptation of the human diaphragm in chronic obstructive pulmonary disease. Eur Respir J 1999; 13: 371-378.

24. Cala SJ, Kenyon CM, Ferrigno G, et al. Chest wall and lung volume estimation by optical reflectance motion analysis. J Appl Physiol 1996; 81: 2680-2689.

25. Aliverti A, Dellaca R, Pelosi P, et al. Compartmental analysis of breathing in the supine and prone positions by optoelectronic plethysmography. Ann Biomed Eng 2001; 29: 60-70.

26. Mauro AL, D'Angelo MG, Romei $M$, et al. Abdominal volume contribution to tidal volume as an early indicator of 


\section{Suggested answers}

1. $\mathrm{a}-\mathrm{d}$.

2. c.

3. a. respiratory impairment in Duchenne muscular dystrophy. Eur RespirJ 2010; 35: 1118-1125.

27. Layton AM, Moran SL, Roychoudhury A, et al. Noninvasive measurement of abnormal ventilatory mechanics in amyotrophic lateral sclerosis. Muscle Nerve 2016; 54: 270-276.

28. Boudarham J, Pradon D, Prigent $\mathrm{H}$, et al. Optoelectronic plethysmography as an alternative method for the diagnosis of unilateral diaphragmatic weakness. Chest 2013; 144: 887-895.

29. Harlaar L, Ciet P, van der Ploeg A, et al. Imaging of respiratory muscles in neuromuscular disease: A review. Neuromuscul Disord 2018; 28: 246-256.
30. Dube BP, Dres M, Mayaux J, et al. Ultrasound evaluation of diaphragm function in mechanically ventilated patients: Comparison to phrenic stimulation and prognostic implications. Thorax 2017; 72: 811-818.

31. Sarlabous L, Torres A, Fiz JA, et al. Inspiratory muscle activation increases with copd severity as confirmed by noninvasive mechanomyographic analysis. PLoS One 2017; 12: e0177730.

32. Nierat MC, Dube BP, Llontop C, et al. Measuring ventilatory activity with structured light plethysmography (SLP) reduces instrumental observer effect and preserves tidal breathing variability in healthy and copd. Front Physiol 2017; 8: 316 . 